\title{
Weighted Pluricomplex Energy II
}

\author{
Slimane Benelkourchi \\ Université de Montréal, Pavillon 3744, Rue Jean-Brillant, Montréal, QC, Canada H3C 3J7 \\ Correspondence should be addressed to Slimane Benelkourchi; slimane.benelkourchi@umontreal.ca
}

Received 31 August 2014; Revised 29 December 2014; Accepted 9 January 2015

Academic Editor: Antonin Novotny

Copyright (C) 2015 Slimane Benelkourchi. This is an open access article distributed under the Creative Commons Attribution License, which permits unrestricted use, distribution, and reproduction in any medium, provided the original work is properly cited.

\begin{abstract}
We continue our study of the complex Monge-Ampère operator on the weighted pluricomplex energy classes. We give more characterizations of the range of the classes $\mathscr{E}_{\chi}$ by the complex Monge-Ampère operator. In particular, we prove that a nonnegative Borel measure $\mu$ is the Monge-Ampère of a unique function $\varphi \in \mathscr{E}_{\chi}$ if and only if $\chi\left(\mathscr{E}_{\chi}\right) \subset L^{1}(d \mu)$. Then we show that if $\mu=\left(d d^{c} \varphi\right)^{n}$ for some $\varphi \in \mathscr{E}_{\chi}$ then $\mu=\left(d d^{c} u\right)^{n}$ for some $\varphi \in \mathscr{E}_{\gamma}$, where $f$ is given boundary data. If moreover the nonnegative Borel measure $\mu$ is suitably dominated by the Monge-Ampère capacity, we establish a priori estimates on the capacity of sublevel sets of the solutions. As a consequence, we give a priori bounds of the solution of the Dirichlet problem in the case when the measure has a density in some Orlicz space.
\end{abstract}

\section{Introduction}

Let $\Omega \subset \mathbb{C}^{n}$ be a bounded hyperconvex domain, that is, a connected, bounded open subset of $\mathbb{C}^{n}$, such that there exists a negative plurisubharmonic function $\rho$ such that $\{z \in$ $\Omega ; \rho(z)<-c\} \Subset \Omega$, for all $c>0$. Such a function $\rho$ is called an exhaustion function. We let $\operatorname{PSH}(\Omega)$ denote the cone of plurisubharmonic functions (psh for short) on $\Omega$ and let $\mathrm{PSH}^{-}(\Omega)$ denote the subclass of negative functions.

As known (see $[1,2]$ ), the complex Monge-Ampère operator $\left(d d^{c} \cdot\right)^{n}$ is well defined, as a nonnegative measure, on the set of locally bounded plurisubharmonic functions. Therefore the question of describing the measures which are the Monge-Ampère of bounded psh functions is very important for pluripotential theory, complex dynamic, and complex geometry. This problem has been studied extensively by various authors; see, for example, [2-6] and reference therein. In [7], Cegrell introduced the pluricomplex energy classes $\mathscr{E}_{p}(\Omega)$ and $\mathscr{F}_{p}(\Omega)(p \geq 1)$ on which the complex Monge-Ampère operator is well defined. He proved that a measure $\mu$ is the Monge-Ampère of some function $u \in \mathscr{E}_{p}(\Omega)$ if and only if it satisfies

$$
\begin{array}{r}
\int_{\Omega}(-v)^{p} d \mu \leq \operatorname{Const}\left(\int_{\Omega}(-v)^{p}\left(d d^{c} v\right)^{n}\right)^{p /(n+p)}, \\
\forall v \in \mathscr{E}_{0}(\Omega),
\end{array}
$$

where $\mathscr{E}_{0}(\Omega)$ is the cone of all bounded psh functions $\varphi$ defined on the domain $\Omega$ with finite total Monge-Ampère mass and $\lim _{z \rightarrow \zeta} \varphi(z)=0$, for every $\zeta \in \partial \Omega$. Recently, Åhag et al. in [8] proved that, in the case $p=1$, inequality (1) is equivalent to $\mathscr{E}_{1}(\Omega) \subset L^{1}(d \mu)$. In this note, our first objective is to extend this result by showing that, for all positive number $p$, inequality (1) is equivalent to $\mathscr{E}_{p}(\Omega) \subset L^{p}(d \mu)$. In fact, we prove some more general result. Given a nondecreasing function $\chi: \mathbb{R}^{-} \rightarrow \mathbb{R}^{-}$, we consider the set $\mathscr{E}_{\chi}(\Omega)$ of plurisubharmonic functions of finite $\chi$-weighted MongeAmpère energy and, in some sense, has boundary values zero. These are the functions $u \in \operatorname{PSH}(\Omega)$ for which there exists a decreasing sequence $u_{j} \in \mathscr{E}_{0}(\Omega)$ with limit $u$ and

$$
\sup _{j \in \mathbb{N}} \int_{\Omega}-\chi\left(u_{j}\right)\left(d d^{c} u_{j}\right)^{n}<\infty .
$$

Then we have the following characterization of the image of the complex Monge-Ampère acting in the class $\mathscr{E}_{\chi}(\Omega)$.

Theorem 1. Let $\chi: \mathbb{R}^{-} \rightarrow \mathbb{R}^{-}$be an increasing convex or homogeneous function such that $\chi(-\infty)=-\infty$. The following assertions are equivalent:

(1) there exists a unique function $\varphi \in \mathscr{E}_{\chi}(\Omega)$ such that $\mu=\left(d d^{c} \varphi\right)^{n}$;

(2) $\chi\left(\mathscr{E}_{\chi}(\Omega)\right) \subset L^{1}(d \mu)$. 
Next, we extend our previous result to families of functions having prescribed boundary data. Let $f \in \operatorname{PSH}(\Omega)$ be a maximal psh function. We define the class $\mathscr{E}_{\chi}(f)$ to be the class of psh functions $u$ such that there exists a function $\varphi \in \mathscr{E}_{\chi}(\Omega)$ such that

$$
\varphi(z)+f(z) \leq u(z) \leq f(z), \quad \forall z \in \Omega .
$$

Some particular cases of the classes $\mathscr{E}_{\chi}(f)$ have been studied in $[6,7,9-16]$.

More precisely, we prove the following result.

Theorem 2. Let $\mu$ be a nonnegative measure in $\Omega$, let $\chi$ : $\mathbb{R}^{-} \rightarrow \mathbb{R}^{-}$be an increasing convex or homogeneous function such that $\chi(-\infty)=-\infty$, and let $f$ be a maximal function. Then, if $\mu=\left(d d^{c} u\right)^{n}$ for some $u \in \mathscr{E}_{\chi}(\Omega)$ then there exists a unique function $\varphi \in \mathscr{E}_{\chi}(f)$ such that $\mu=\left(d d^{c} \varphi\right)^{n}$.

Moreover, when the nonnegative measure $\mu$ is dominated by the Monge-Ampère capacity, we give an estimate of the growth of solutions of the equation $\left(d d^{c} \varphi\right)^{n}=\mu$. As in [12], let us consider the function

$$
F_{\mu}(t):=\sup \left\{\mu(K), K \Subset \Omega ; \operatorname{cap}_{\Omega}(K) \leq t\right\}, \quad \forall t \geq 0 .
$$

Then $F:=F_{\mu}$ is a nondecreasing function on $\mathbb{R}^{+}$and satisfies

$$
\mu(K) \leq F_{\mu}\left(\operatorname{cap}_{\mu}(K)\right), \quad \forall \text { Borel subsets } K \subset \Omega \text {. }
$$

Write $F(x)=F_{\varepsilon}(x)=x(\varepsilon(-\ln (x / n)))^{n}$ where $\varepsilon: \mathbb{R}^{+} \rightarrow \mathbb{R}^{+}$ is nondecreasing.

Such measures dominated by the Monge-Ampère capacity have been extensively studied by Kołodziej in [3-5]. He proved that if $\phi: \partial \Omega \rightarrow \mathbb{R}$ is a continuous function and $\int_{0}^{+\infty} \varepsilon(t) d t<+\infty$, then $\mu$ is the Monge-Ampère of a unique function $\varphi \in \operatorname{PSH}(\Omega)$ with $\varphi_{/ \partial \Omega}=\phi$.

When $\int_{0}^{+\infty} \varepsilon(s) d s=+\infty$, we have the following estimate.

Theorem 3. Let $\mu$ be a positive finite measure. Assume, for all compact subsets $K \subset \Omega$,

$$
\mu(K) \leq F_{\varepsilon}\left(\operatorname{cap}_{\Omega}(K)\right) .
$$

Then there exists a unique function $\varphi \in \mathscr{F}(f)$ such that $\mu=$ $\left(d d^{c} \varphi\right)^{n}$, and

$$
\operatorname{cap}_{\Omega}(\{\varphi<f-s\}) \leq \exp \left(-n H^{-1}(s)\right), \quad \forall s>0 .
$$

Here $H^{-1}$ is the reciprocal function of $H(x)=e \int_{0}^{x} \varepsilon(t) d t+$ $s_{0}(\mu)$.

$$
\begin{aligned}
& \text { In particular if } \int_{0}^{+\infty} \varepsilon(t) d t<+\infty \text { then } \\
& \quad 0 \leq f-\varphi \leq e \int_{0}^{+\infty} \varepsilon(t) d t+e \varepsilon(0)+\mu(\mu)^{1 / n} .
\end{aligned}
$$

The paper is organised as follows. In Section 2, we recall the definitions of the energy classes $\mathscr{E}_{\chi}(\Omega)$ and some classes of psh functions introduced by Cegrell $[7,13,14]$ and we prove Theorem 1. In Section 3, we prove Theorem 2. As a consequence, we generalize the main result in the paper [9]. In Section 4, we prove Theorem 3. As application, we give a priori bound of the solution of Dirichlet problem in the case when the measure $\mu=g d \lambda$, where $g$ belongs to some Orlicz space $L \log ^{\alpha} L$.

\section{Energy Classes with Zero Boundary Data $\mathscr{E}_{\chi}$}

Let us recall some Cegrell's classes (Cf. $[7,13,14])$. The class $\mathscr{E}(\Omega)$ is the set of plurisubharmonic functions $u$ such that, for all $z_{0} \in \Omega$, there exists a neighbourhood $V_{z_{0}}$ of $z_{0}$ and $u_{j} \in \mathscr{E}_{0}(\Omega)$, a decreasing sequence which converges towards $u$ in $V_{z_{0}}$ and satisfies $\sup _{j} \int_{\Omega}\left(d d^{c} u_{j}\right)^{n}<+\infty$. Cegrell [13] has shown that the operator $\left(d d^{c} \cdot\right)^{n}$ is well defined on $\mathscr{E}(\Omega)$, continuous under decreasing limits, and the class $\mathscr{E}(\Omega)$ is stable under taking maximum; that is, if $u \in \mathscr{E}(\Omega)$ and $v \in \operatorname{PSH}^{-}(\Omega)$ then $\max (u, v) \in \mathscr{E}(\Omega)$. This class is the largest class with these properties (Theorem 4.5 in [13]). The class $\mathscr{E}(\Omega)$ has been further characterized by Błocki $[17,18]$ and Le Mau et al. in [19].

The class $\mathscr{F}(\Omega)$ is the global version of $\mathscr{E}(\Omega)$ : a function $u$ belongs to $\mathscr{F}(\Omega)$ if and only if there exists a decreasing sequence $u_{j} \in \mathscr{E}_{0}(\Omega)$ converging towards $u$ in all of $\Omega$, which satisfies $\sup _{j} \int_{\Omega}\left(d d^{c} u_{j}\right)^{n}<+\infty$. The class $\mathscr{E}(\Omega)$ has been further characterized in $[12,17]$.

Let $\Omega_{j} \Subset \Omega$ be an increasing sequence of strictly pseudoconvex domains such that $\Omega=\bigcup_{j} \Omega_{j}$. Let $u \in \mathscr{E}(\Omega)$ be a given psh function and put

$$
u_{\Omega_{j}}:=\sup \left\{\varphi \in \operatorname{PSH}(\Omega) ; \varphi \leq u \text { on } \Omega \backslash \Omega_{j}\right\} .
$$

Then we have $u_{\Omega_{j}} \in \mathscr{E}(\Omega)$ and $u_{\Omega_{j}}$ is an increasing sequence. Let $\tilde{u}:=\left(\lim _{j} u_{\Omega_{j}}\right)^{*}$. It follows from the properties of $\mathscr{E}(\Omega)$ that $\tilde{u} \in \mathscr{E}(\Omega)$. Note that the definition of $\tilde{u}$ is independent of the choice of the sequence $\Omega_{j}$ and is maximal; that is, $\left(d d^{c} \widetilde{u}\right)^{n}=0 . \tilde{u}$ is the smallest maximal psh function above $u$. Define $\mathcal{N}(\Omega):=\{u \in \mathscr{E}(\Omega) ; \tilde{u}=0\}$. In fact, this class is the analogous of potentials for subharmonic functions.

Definition 4. Let $\chi: \mathbb{R}^{-} \rightarrow \mathbb{R}^{-}$be a nondecreasing function. We let $\mathscr{E}_{\chi}(\Omega)$ denote the set of all functions $u \in \operatorname{PSH}(\Omega)$ for which there exists a sequence $u_{j} \in \mathscr{E}_{0}(\Omega)$ decreasing to $u$ in $\Omega$ and satisfying

$$
\sup _{j \in \mathbb{N}} \int_{\Omega}-\chi\left(u_{j}\right)\left(d d^{c} u_{j}\right)^{n}<\infty .
$$

It was proved in $[15,20]$ that if $\chi \not \equiv 0$ then

$$
\mathscr{E}_{\chi}(\Omega) \subset \mathscr{E}(\Omega) .
$$

In particular, for any function $u \in \mathscr{E}_{\chi}(\Omega)$, the complex Monge-Ampère operator $\left(d d^{c} u\right)^{n}$ is well defined as nonnegative measure. Furthermore, if $\chi(-t)<0$ for all $t>0$, then

$$
\mathscr{E}_{\chi}(\Omega)=\left\{u \in \mathcal{N}(\Omega) ; \int_{\Omega}-\chi(u)\left(d d^{c} u\right)^{n}<+\infty\right\} .
$$


The class $\mathscr{E}_{\chi}(\Omega)$ has been characterized by the speed of decrease of the capacity of sublevel sets $[11,12]$.

Recall that the Monge-Ampère capacity has been introduced and studied by Bedford and Taylor in [1]. Given $K \subset \Omega$, a compact subset, its Monge-Ampère capacity relatively to $\Omega$ is defined by

$$
\operatorname{cap}_{\Omega}(K):=\sup \left\{\int_{K}\left(d d^{c} u\right)^{n} ; u \in \operatorname{PSH}(\Omega),-1 \leq u \leq 0\right\} .
$$

The following estimates (cf [12]) will be useful later on. For any $\varphi \in \mathscr{E}_{0}$

$$
\begin{aligned}
& t^{n} \operatorname{cap}_{\Omega}(\varphi<-s-t) \\
& \quad \leq \int_{(\varphi<-s)}\left(d d^{c} \varphi\right)^{n} \leq s^{n} \operatorname{cap}_{\Omega}(\varphi<-s), \quad \forall s, t>0 .
\end{aligned}
$$

Let $\chi: \mathbb{R}^{-} \rightarrow \mathbb{R}^{-}$be a nondecreasing function. Without loss of generality, from now on, we assume that $\chi(0)=0$. We define the class $\widehat{\mathscr{E}}_{\chi}(\Omega)$

$$
\begin{aligned}
\widehat{\mathscr{E}}_{\chi}(\Omega):= & \left\{\varphi \in \operatorname{PSH}^{-}(\Omega) ;\right. \\
& \left.\int_{t_{\chi}}^{+\infty} t^{n} \chi^{\prime}(t) \operatorname{cap}_{\Omega}(\varphi<-t) d t<+\infty\right\} .
\end{aligned}
$$

Proposition 5. One has $\widehat{\mathscr{E}}_{\chi}(\Omega) \subset \mathscr{E}_{\chi}(\Omega)$, while

$$
\mathscr{E}_{\chi}(\Omega) \subset \widehat{\mathscr{E}}_{\hat{\chi}}(\Omega), \quad \text { where } \hat{\chi}(t)=\chi\left(\frac{t}{2}\right) .
$$

Moreover, if $\chi:]-\infty,-t_{\chi}\left[\rightarrow \mathbb{R}^{-}\right.$is convex, then

$$
\mathscr{E}_{\chi}(\Omega)=\widehat{\mathscr{E}}_{\chi}(\Omega) \text {. }
$$

Here $t_{\chi}$ denote the real number satisfying $\chi(t)<0$, for all $t<$ $-t_{\chi}$, and $\chi(t)=0$, for all $t \geq-t_{\chi}$.

Proof. Compare with $[12,20]$.

Theorem 6. Let $\chi: \mathbb{R}^{-} \rightarrow \mathbb{R}^{-}$be an increasing convex function such that $\chi(-\infty)=-\infty$. The following conditions are equivalent:

(1) there exists a unique function $\varphi \in \mathscr{E}_{\chi}(\Omega)$ such that $\mu=\left(d d^{c} \varphi\right)^{n}$

(2) $\chi\left(\mathscr{E}_{\chi}(\Omega)\right) \subset L^{1}(\Omega, d \mu)$;

(3) there exists a constant $C>0$ such that

$$
\int_{\Omega}-\chi(u) d \mu \leq C, \quad \forall u \in \widetilde{\mathscr{E}}_{0}(\Omega) ;
$$

(4) there exists a constant $A>0$ such that

$$
\begin{aligned}
& \int_{\Omega}-\chi(u) d \mu \\
& \quad \leq C_{2} \max \left(1,\left(\int_{0}^{\infty} s^{n} \chi^{\prime}(-s) c a p_{\Omega}(u<-s) d s\right)^{1 / n}\right),
\end{aligned}
$$

(5) there exists a locally bounded function $F: \mathbb{R}^{+} \rightarrow \mathbb{R}^{+}$ such that $\limsup _{t \rightarrow+\infty} F(t) / t<1$ and

$$
\int_{\Omega}-\chi(u) d \mu \leq F\left(C_{\chi}(u)\right), \quad \forall u \in \mathscr{E}_{0}(\Omega) .
$$

Here $\widetilde{\mathscr{E}}_{0}(\Omega)$ denotes the class $\widetilde{\mathscr{E}}_{0}(\Omega):=\left\{u \in \mathscr{E}_{0}(\Omega)\right.$; $\left.\int_{0}^{\infty} s^{n} \chi^{\prime}(-s) \operatorname{cap}_{\Omega}(u<-s) d s \leq 1\right\}$ and $C_{\chi}(u):=$ $\int_{0}^{\infty} s^{n} \chi^{\prime}(-s) c a p_{\Omega}(u<-s) d s$.

The equivalences (1) $\Leftrightarrow(3) \Leftrightarrow$ (4) are proved in [11] (Theorem 5.1) and the implication (5) $\Rightarrow$ (1) is proved in [12] (Theorem 5.2). For the sake of completeness we include a complete proof.

Proof. We start by the implication (1) $\Rightarrow$ (2). Let $u, \varphi \in$ $\mathscr{E}_{\chi}(\Omega)$. It follows from Proposition 5 that $u+\varphi \in \mathscr{E}_{\chi}(W)$. Hence

$$
\int_{\Omega}-\chi(u)\left(d d^{c} \varphi\right)^{n} \leq \int_{\Omega}-\chi(u+\varphi)\left(d d^{c}(u+\varphi)\right)^{n}<\infty .
$$

Now, for the implication (2) $\Rightarrow(3)$, assume that (3) is not satisfied. Then for each $j \in \mathbb{N}$ we can find a function $u_{j} \in$ $\widetilde{\mathscr{E}}_{0}(\Omega)$ such that

$$
\int_{\Omega}-\chi\left(u_{j}\right) d \mu \geq 2^{3 j} .
$$

Consider the function

$$
u:=\sum_{j=1}^{\infty} \frac{1}{2^{2 j}} u_{j} .
$$

Observe that

$$
(u<-s) \subset \bigcup_{1}^{\infty}\left(u_{j}<-2^{j} s\right) .
$$

Hence

$$
\operatorname{cap}_{\Omega}(u<-s) \leq \sum_{j}^{\infty} \operatorname{cap}_{\Omega}\left(u_{j}<-2^{j} s\right)
$$

Now, since the weight $\chi$ is convex or homogeneous and using the estimates (14), we get

$$
\begin{aligned}
& \int_{0}^{\infty} s^{n} \chi^{\prime}(-s) \operatorname{cap}_{\Omega}(u<-s) d s \\
& \quad \leq \int_{0}^{\infty} s^{n} \chi^{\prime}(-s) \sum_{j}^{\infty} \operatorname{cap}_{\Omega}\left(u_{j}<-2^{j} s\right) d s \\
& \leq \sum_{j}^{\infty} \frac{1}{2^{n j-n}} \int_{0}^{\infty}\left(2^{j-1} s\right)^{n} \chi^{\prime}(-s) \operatorname{cap}_{\Omega}\left(u_{j}<-2^{j} s\right) \\
& \leq \sum_{j}^{\infty} \frac{1}{2^{n j-n}} \int_{0}^{\infty}\left(2^{j-1} s\right)^{n} \chi^{\prime}(-s) \operatorname{cap}_{\Omega}\left(u_{j}<-s\right) \\
& \leq 2^{n} \sum_{j}^{\infty} \frac{1}{2^{n j}}<\infty .
\end{aligned}
$$


Hence $u \in \mathscr{E}_{\chi}(\Omega)$. On the other hand, from (22) we have

$$
\int_{\Omega}-\chi(u) d \mu \geq \frac{1}{2^{2 j}} \int_{\Omega}-\chi\left(u_{j}\right) d \mu \geq 2^{j}, \quad \forall j \in \mathbb{N},
$$

which yields a contradiction.

Now, we prove that $(3) \Rightarrow(4)$. Let $\psi \in \mathscr{E}_{0}(\Omega)$, denote $E_{\chi}(\psi):=\int_{\Omega}-\chi(\psi)\left(d d^{c} \psi\right)^{n}$. If $\psi \in \widetilde{\mathscr{E}}_{0}(\Omega)$, that is, $C_{\chi}(\psi) \leq 1$, then

$$
\int_{\Omega}-\chi(\psi) d \mu \leq 2^{n}=C .
$$

If $C_{\chi}(\psi)>1$ the function $\tilde{\psi}$ defined by

$$
\widetilde{\psi}:=\frac{\psi}{1+C_{\chi}(\psi)^{1 / n}} \in \widetilde{\mathscr{E}}_{0}(\Omega) .
$$

Indeed, from the monotonicity of $\chi$, we have

$$
\begin{gathered}
\int_{0}^{\infty} \chi^{\prime}(s) s^{n} \operatorname{cap}_{\Omega}\left(\frac{\psi}{1+C_{\chi}(\psi)^{1 / n}}<-s\right) d s \\
=\frac{1}{C_{\chi}(\psi)} \int_{0}^{\infty} \chi^{\prime}(s)\left(s C_{\chi}(\psi)^{1 / n}\right)^{n} \\
\cdot \operatorname{cap}_{\Omega}\left(\psi<-s-s C_{\chi}(\psi)^{1 / n}\right) d s \\
\leq \frac{1}{C_{\chi}(\psi)} \int_{0}^{\infty} \chi^{\prime}(s) s^{n} \operatorname{cap}_{\Omega}(\psi<-s) d s=1 .
\end{gathered}
$$

It follows from (18) and the convexity of $\chi$ that

$$
\begin{aligned}
\int_{\Omega}-\chi(\psi) d \mu & \leq 2 C_{\chi}(\psi)^{1 / n} \int_{\Omega}-\chi\left(\frac{\psi}{1+C_{\chi}(\psi)^{1 / n}}\right) d \mu \\
& \leq A C_{\chi}(\psi)^{1 / n} .
\end{aligned}
$$

Hence we get (19).

For the implication (4) $\Rightarrow(5)$, we consider $F(t)=$ $A \max \left(1, t^{1 / n}\right)$.

$(5) \Rightarrow(1)$. It follows from [12] (Theorem 4.5) that the class $\mathscr{E}_{\chi}(\Omega)$ characterizes pluripolar sets in the sense that if $P$ is a locally pluripolar subset of $\Omega$ then $P \subset\{v=-\infty\}$, for some $v \in \mathscr{E}_{\chi}(\Omega)$. Then assumption (20) on $\mu$ implies that it vanishes on pluripolar sets. It follows from [13] that there exists a function $u \in \mathscr{E}_{0}(\Omega)$ and $f \in L_{\text {loc }}^{1}\left(\left(d d^{c} u\right)^{n}\right)$ such that $\mu=f\left(d d^{c} u\right)^{n}$.

Consider $\mu_{j}:=\min (f, j)\left(d d^{c} u\right)^{n}$. This is a finite measure which is bounded from above by the complex Monge-Ampère measure of a bounded function. It follows therefore from [3] that there exist $\varphi_{j} \in \mathscr{E}_{0}(\Omega)$ such that

$$
\left(d d^{c} \varphi_{j}\right)^{n}=\min (f, j)\left(d d^{c} u\right)^{n} .
$$

The comparison principle shows that $\varphi_{j}$ is a decreasing sequence. Set $\varphi=\lim _{j \rightarrow \infty} \varphi_{j}$. It follows from (20) that

$$
\int_{\Omega}-\chi\left(\varphi_{j}\right)\left(d d^{c} \varphi_{j}\right)^{n} \leq F\left(\int_{0}^{\infty} \chi^{\prime}(s) s^{n} \operatorname{cap}_{\Omega}\left(\varphi_{j}<-s\right) d s\right) .
$$

Hence

$$
\sup _{j} \int_{0}^{\infty} \chi^{\prime}(s) s^{n} \operatorname{cap}_{\Omega}\left(\varphi_{j}<-s\right) d s<\infty .
$$

This implies that

$$
\int_{0}^{+\infty} t^{n} \chi^{\prime}(-t) \operatorname{cap}_{\Omega}(\{\varphi<-t\}) d t<+\infty
$$

Then $\varphi \not \equiv-\infty$ and therefore $\varphi \in \mathscr{E}_{\chi}(\Omega)$.

We conclude now by continuity of the complex MongeAmpère operator along decreasing sequences that $\left(d d^{c} \varphi\right)^{n}=$ $\mu$. The uniqueness of $\varphi$ follows from the comparison principle.

\section{The Weighted Energy Class with Boundary Values}

Let $\chi: \mathbb{R}^{-} \rightarrow \mathbb{R}^{-}$be a nondecreasing function and let $f \in$ $\mathscr{M}(\Omega)$ be a maximal psh function. We define the class $\mathscr{E}_{\chi}(f)$ (resp., $\mathcal{N}(f), \mathscr{F}(f), \mathscr{N}^{a}(f)$, and $\left.\mathscr{F}^{a}(f)\right)$ to be the class of psh functions $u$ such that there exists a function $\varphi \in \mathscr{E}_{\chi}(\Omega)$ (resp., $\mathcal{N}, \mathscr{F}, \mathcal{N}^{a}, \mathscr{F}^{a}$ ) such that

$$
\varphi(z)+f(z) \leq u(z) \leq f(z), \quad \forall z \in \Omega .
$$

Later on, we will use repeatedly the following well known comparison principle from [1] as well as its generalizations to the class $\mathscr{N}(f)$ (cf. $[10,14])$.

Theorem 7 (see $[1,10,14]$ ). Let $f \in \mathscr{E}(\Omega)$ be a maximal function and $u, v \in \mathcal{N}(f)$ be such that $\left(d d^{c} u\right)^{n}$ vanishes on all pluripolar sets in $\Omega$. Then

$$
\int_{(u<v)}\left(d d^{c} v\right)^{n} \leq \int_{(u<v)}\left(d d^{c} u\right)^{n} .
$$

Furthermore if $\left(d d^{c} u\right)^{n}=\left(d d^{c} v\right)^{n}$ then $u=v$.

The following lemma, which gives an estimate of the size of sublevel set in terms of the mass of Monge-Ampère measure, will be useful shortly.

Lemma 8. Let $\chi: \mathbb{R}^{-} \rightarrow \mathbb{R}^{-}$be a nondecreasing function such that $\chi(t)<0$, for all $t<0$, and $f \in \mathscr{E}$ a maximal function. Then for all $\varphi \in \mathscr{E}_{\chi}(f)$

$$
\begin{aligned}
& t^{n} \operatorname{cap}_{\Omega}(\varphi<-s-t+f) \\
& \quad \leq \int_{(\varphi<-s+f)}\left(d d^{c} \varphi\right)^{n}, \quad \forall s>0, t>0 .
\end{aligned}
$$

Proof. Fix $s, t>0$. Let $K \subset\{\varphi<f-s-t\}$ be a compact subset. Then

$$
\begin{aligned}
\operatorname{cap}_{\Omega}(K) & =\int_{\Omega}\left(d d^{c} u_{K}^{*}\right)^{n}=\int_{(\varphi<f-s-t)}\left(d d^{c} u_{K}^{*}\right)^{n} \\
& =\int_{\left(\varphi<f-s+t u_{K}^{*}\right)}\left(d d^{c} u_{K}^{*}\right)^{n} \leq \frac{1}{t^{n}} \int_{\{\varphi<v\}}\left(d d^{c} v\right)^{n},
\end{aligned}
$$


where $u_{K}^{*}$ is the relative extremal function of the compact $K$ and $v:=f-s+t u_{K}^{*}$. It follows from Theorem 7 that

$$
\begin{aligned}
\frac{1}{t^{n}} \int_{\{\varphi<v\}}\left(d d^{c} v\right)^{n} & =\frac{1}{t^{n}} \int_{\{\varphi<\max (\varphi, v)\}}\left(d d^{c} \max (\varphi, v)\right)^{n} \\
& \leq \frac{1}{t^{n}} \int_{\{\varphi<\max (\varphi, v)\}}\left(d d^{c} \varphi\right)^{n} \\
& \left.=\frac{1}{t^{n}} \int_{\left\{\varphi<f-s+t u_{K}\right\}}\left(d d^{c} \varphi\right)\right)^{n} \\
& \left.\leq \frac{1}{t^{n}} \int_{\{\varphi<f-s\}}\left(d d^{c} \varphi\right)\right)^{n} .
\end{aligned}
$$

Taking the supremum over all $K$ 's yields the first inequality.

Proposition 9. Let $\chi: \mathbb{R}^{-} \rightarrow \mathbb{R}^{-}$be an increasing function. Then one has

$$
\begin{aligned}
\mathscr{E}_{\chi}(f) \subset\{u \in \operatorname{PSH}(\Omega) ; u \leq f, \\
\\
\left.\qquad \int_{0}^{+\infty} s^{n} \chi^{\prime}(-s) \operatorname{cap}_{\Omega}(u<f-2 s) d s<+\infty\right\} .
\end{aligned}
$$

In particular, if $\chi \neq \equiv 0$, then $\operatorname{cap}_{\Omega}(u<f-s)<+\infty$ for all $s>0$ and $u \in \mathscr{E}_{\chi}(f)$.

Proof. Let $u \in \mathscr{E}_{\chi}(f)$. Then there exists a function $\varphi \in \mathscr{E}_{\chi}(\Omega)$ such that $\varphi+f \leq u$. Therefore $(u<f-s) \subset(\varphi<-s)$. It follows from Lemma 8

$$
\begin{gathered}
\int_{0}^{+\infty} s^{n} \chi^{\prime}(-s) \operatorname{cap}_{\Omega}(u<f-2 s) d s \\
\quad \leq \int_{0}^{+\infty} s^{n} \chi^{\prime}(-s) \operatorname{cap}_{\Omega}(\varphi<-2 s) d s \\
\quad \leq \int_{0}^{+\infty} \chi^{\prime}(-s) \int_{(\varphi<-s)}\left(d d^{c} \varphi\right)^{n} d s \\
\quad=\int_{\Omega}-\chi(\varphi)\left(d d^{c} \varphi\right)^{n}<\infty .
\end{gathered}
$$

Theorem 10. Let $\chi: \mathbb{R}^{-} \rightarrow \mathbb{R}^{-}$be an increasing function which satisfies $\chi(-\infty)=-\infty$ and $f \in \mathscr{E}$ a maximal function. Then if there exists a decreasing sequence $u_{j} \in \mathscr{E}_{0}(f)$ such that

$$
\sup _{j} \int_{\Omega}-\chi\left(u_{j}-f\right)\left(d d^{c} u_{j}\right)^{n}<\infty
$$

then $u:=\lim _{j \rightarrow \infty} u_{j} \in \mathscr{E}_{\chi}(f)$ and $\chi(u-f) \in L^{1}\left(\left(d d^{c} u\right)^{n}\right)$.

Conversely, if $u \in \mathscr{E}_{\chi}(f)$ and $\chi(u-f) \in L^{1}\left(\left(d d^{c} u\right)^{n}\right)$ then there exists sequence $u_{j} \in \mathscr{E}_{0}(f)$ decreasing towards $u$ such that

$$
\sup _{j} \int_{\Omega}-\chi\left(u_{j}-f\right)\left(d d^{c} u_{j}\right)^{n}<\infty
$$

Proof. Assume that the sequence $u_{j} \in \mathscr{E}_{0}(f) \cap C(\Omega)$ (if necessary, we approximate $u_{j}$ by a continuous sequence $u_{j}^{k} \epsilon$ $\left.\mathscr{E}_{0}(f)\right)$. For a fixed $j \in \mathbb{N}$, let $\varphi_{j}$ denote the function defined by

$$
\varphi_{j}(z):=\sup \left\{v \in \operatorname{PSH}(\Omega) ; v+f \leq u_{j}\right\}, \quad \forall z \in \Omega .
$$

We claim that

(1) $\varphi_{j} \in \mathscr{E}_{0}$;

(2) $\left(d d^{c} \varphi_{j}\right)^{n} \leq\left(d d^{c} u_{j}\right)^{n}$

(3) $\left(d d^{c} \varphi_{j}\right)^{n}=0$ on the subset $\left(\varphi_{j}+f<u_{j}\right)$.

Then, it follows from statements (1), (2), and (3) that for each $j \in \mathbb{N}$

$$
\begin{aligned}
\int_{\Omega}-\chi\left(\varphi_{j}\right)^{n}\left(d d^{c} \varphi_{j}\right)^{n} & =\int_{\left(\varphi_{j}+f=u_{j}\right)}-\chi\left(\varphi_{j}\right)^{n}\left(d d^{c} \varphi_{j}\right)^{n} \\
& \leq \int_{\Omega}-\chi\left(u_{j}-f\right)^{n}\left(d d^{c} u_{j}\right)^{n}
\end{aligned}
$$

Therefore

$$
\begin{aligned}
& \sup _{j} \int_{\Omega}-\chi\left(\varphi_{j}\right)^{n}\left(d d^{c} \varphi_{j}\right)^{n} \\
& \quad \leq \sup _{j} \int_{\Omega}-\chi\left(u_{j}-f\right)^{n}\left(d d^{c} u_{j}\right)^{n}<+\infty .
\end{aligned}
$$

Hence, the function $\varphi:=\lim _{j \rightarrow \infty} \varphi_{j}$ satisfies $\varphi+f \leq u \leq f$.

For the converse implication, fix $u \in \mathscr{E}_{\chi}(f)$. Then there exists a function $\varphi \in \mathscr{E}_{\chi}$ such that $\varphi+f \leq u \leq f$. Let $\varphi_{j} \in$ $\mathscr{E}_{0} \cap C(\Omega)$ be a decreasing sequence with limit function $\varphi$. Then for each $j \in \mathbb{N}$, consider the function $u_{j}:=\max \left(\varphi_{j}+\right.$ $f, u) \in \mathscr{E}_{\chi}(f)$. The sequence $u_{j}$ decreases towards $u$ and

$$
\begin{aligned}
& \int_{\Omega}-\chi\left(u_{j}-f\right)^{n}\left(d d^{c} u_{j}\right)^{n} \\
& \quad \leq C \int_{\Omega}-\chi(u-f)^{n}\left(d d^{c} u\right)^{n}<\infty,
\end{aligned}
$$

where $C$ is a constant which depends only on $u$ and the proof of the theorem is completed.

Theorem 11. Let $\mu$ be a nonnegative measure in $\Omega$, let $\chi$ : $\mathbb{R}^{-} \rightarrow \mathbb{R}^{-}$be an increasing convex (or homogeneous) function such that $\chi(-\infty)=-\infty$, and let $f \in \mathscr{E}(\Omega)$ be a maximal function. Then there exists a unique function $\varphi \in \mathscr{E}_{\chi}(f)$ such that $\mu=\left(d d^{c} \varphi\right)^{n}$ if and only if $\mu$ satisfies one of the conditions of Theorem 6 .

Proof. Assume that $\mu=\left(d d^{c} v\right)^{n}$ for some $v \in \mathscr{E}_{\chi}$. Let $\left(\Omega_{j}\right)_{j}$ be a fundamental sequence of strictly pseudoconvex subsets of $\Omega$. Choose a sequence $f_{j} \in \operatorname{PSH}(\Omega) \cap C(\bar{\Omega})$ decreasing towards $f$ on $\Omega$ and $f_{j}$ is maximal on $\Omega_{j+1}$. It follows from [13] that there exist a function $g \in \mathscr{E}_{0}$ and a function $\theta \in$ $L_{\text {loc }}^{1}\left(d d^{c} g\right)^{n}$ such that

$$
\mu=\theta\left(d d^{c} g\right)^{n}
$$


Consider the measure $\mu_{j}=\nVdash_{\Omega_{j}} \min (\theta, j)\left(d d^{c} g\right)^{n}$, where $\nVdash_{\Omega_{j}}$ denotes the characteristic function of the set $\Omega_{j}$. Now, solving the Dirichlet problem in the strictly pseudoconvex domain $\Omega_{j}$, we state that there exist functions $u_{j}, v_{j} \in \operatorname{PSH}\left(\Omega_{j}\right) \cap$ $C\left(\bar{\Omega}_{j}\right)$ such that

$$
\begin{array}{r}
\left(d d^{c} u_{j}\right)^{n}=\left(d d^{c} v_{j}\right)^{n}=\mu_{j}, \quad v_{j}=0, \quad u_{j}=f_{j} \\
\text { on } \partial \Omega_{j} .
\end{array}
$$

By the comparison principle, we have $u_{j}$ and $v_{j}$ are decreasing sequences and

$$
v+f \leq v_{j}+f_{j} \leq u_{j} \leq f_{j} \quad \text { on } \Omega_{j} .
$$

Letting $j \rightarrow+\infty$ we get that $u:=\lim _{j \rightarrow \infty} u_{j} \in \mathscr{E}_{\chi}(f)$. The continuity of the complex Monge-Ampère operator under monotonic sequences yields that $\left(d d^{c} u\right)^{n}=\mu$. Uniqueness of $u$ follows from the comparison principle.

Corollary 12. Let $\mu$ be nonnegative measure in $\Omega$ with total finite mass $\mu(\mu)<\infty$ and let $f$ be a maximal function. Then there exists a uniquely determined function $\varphi \in \mathscr{F}^{a}(f)$ such that $\left(d d^{c} \varphi\right)^{n}=\mu$ if and only if $\mu$ vanishes on pluripolar subsets.

Proof. It follows from [13] that there exist a function $\psi \in \mathscr{E}_{0}$ and a function $\theta \in L_{\text {loc }}^{1}\left(d d^{c} \psi\right)^{n}$ such that

$$
\mu=\theta\left(d d^{c} \psi\right)^{n} .
$$

By [3], there exists a unique $h_{j} \in \mathscr{E}_{0}$ such that $\left(d d^{c} h_{j}\right)^{n}=$ $\min (\theta, j)\left(d d^{c} \psi\right)^{n}$. The comparison principle yields that $h_{j}$ is a decreasing sequence. Let denote by $h:=\lim _{j \rightarrow \infty} h_{j}$. It follows from Lemma 8 that $h \neq \equiv-\infty$. Therefore $h \in \mathscr{F}^{a}$. By the continuity of the complex Monge-Ampère operator under decreasing sequences, we have $\left(d d^{c} h\right)^{n}=\mu$. Now, since

$$
\mathscr{F}^{a}=\bigcup_{\substack{\chi \text { convex; } \chi(0) \neq 0 \\ \chi(-\infty)=-\infty}} \mathscr{E}_{\chi},
$$

then there exists a convex function $\chi: \mathbb{R}^{-} \rightarrow \mathbb{R}^{-}$with $\chi(0) \neq$ 0 and $\chi(-\infty)=-\infty$ such that $h \in \mathscr{E}_{\chi}$. By Theorem 11, we can find a unique function $\varphi \in \mathscr{E}_{\chi}(f) \subset \mathscr{F}^{a}(f)$ such that $\left(d d^{c} \varphi\right)^{n}=\mu$.

\section{Measures Dominated by Capacity}

Throughout this section, $\mu$ denotes a fixed nonnegative measure of finite total mass $\mu(\Omega)<+\infty$. We want to solve the Dirichlet problem

$$
\left(d d^{c} \varphi\right)^{n}=\mu, \quad \text { with } \varphi \in \mathscr{F}^{a}(f), \quad \varphi_{\mid \partial \Omega}=f,
$$

and measure how far the distance between the solution $\varphi$ and the given boundary data $f$ is from being bounded, by assuming that $\mu$ is suitable dominated by the Monge-Ampère capacity.

Measures dominated by the Monge-Ampère capacity have been extensively studied by Kołodziej in [3-5]. The main result of his study, achieved in [4], can be formulated as follows. Fix $\varepsilon: \mathbb{R} \rightarrow[0,+\infty[$ a continuous decreasing function and set $F_{\varepsilon}(x):=x[\varepsilon(-\ln x / n)]^{n}$. If for all compact subsets $K \subset \Omega$

$$
\mu(K) \leq F_{\varepsilon}\left(\operatorname{cap}_{\Omega}(K)\right), \quad \text { where } \int_{0}^{+\infty} \varepsilon(t) d t<+\infty,
$$

and $l: \partial \Omega \rightarrow \mathbb{R}$ is a continuous function, then $\mu=\left(d d^{c} \varphi\right)^{n}$ for some continuous function $\varphi \in \operatorname{PSH}(\Omega)$ with $\varphi_{\mid \partial \Omega}=l$.

The condition $\int_{0}^{+\infty} \varepsilon(t) d t<+\infty$ means that $\varepsilon$ decreases fast enough towards zero at infinity. This gives a quantitative estimate on how fast $\varepsilon\left(-\ln \operatorname{Cap}_{\Omega}(K) / n\right)$, hence $\mu(K)$ decreases towards zero as $\operatorname{cap}_{\Omega}(K) \rightarrow 0$.

When $\int_{0}^{+\infty} \varepsilon(t) d t=+\infty$, it is still possible to show that $\mu=\left(d d^{c} \varphi\right)^{n}$ for some function $\varphi \in \mathscr{F}(\Omega)$, but $\varphi$ will generally be unbounded. We now measure how far it is from being so.

Theorem 13. Let $\mu$ be a nonnegative finite measure. Assume for all compact subsets $K \subset \Omega$

$$
\mu(K) \leq F_{\varepsilon}\left(\operatorname{cap}_{\Omega}(K)\right) .
$$

Then there exists a unique function $\varphi \in \mathscr{F}^{a}(f)$ such that $\mu=$ $\left(d d^{c} \varphi\right)^{n}$, and

$$
\operatorname{cap}_{\Omega}(\{\varphi<f-s\}) \leq \exp \left(-n H^{-1}(s)\right), \quad \forall s>0 .
$$

Here $H^{-1}$ is the reciprocal function of $H(x)=e \int_{0}^{x} \varepsilon(t) d t+$ $e \varepsilon(0)+\mu(\Omega)^{1 / n}$.

The proof is almost the same as that of Theorem 5.1 in [12], except that we use Corollary 12 for the existence of the solution and Lemma 8 to estimate the capacity of sublevel set.

Observe that if $\int^{\infty} \varepsilon(t) d t<\infty$ then $H$ is bounded by $e \int_{0}^{\infty} \varepsilon(t) d t+e \varepsilon(0)+\mu(\Omega)^{1 / n}$. Hence $H^{-\infty}(t)=+\infty, \forall t \geq$ $e \int_{0}^{\infty} \varepsilon(t) d t+e \varepsilon(0)+\mu(\Omega)^{1 / n}$. Therefore

$$
0 \leq f-\varphi \leq e \int_{0}^{\infty} \varepsilon(t) d t+e \varepsilon(0)+\mu(\Omega)^{1 / n} .
$$

Now, we consider the case when $\mu=f d \lambda$ is absolutely continuous with respect to Lebesgue measure.

Let $\mathbb{G} \subset \mathbb{C}^{n}$ denote a generic subspace of $\mathbb{C}^{n}$ that is a real subspace such that $\mathbb{G}+J \mathbb{G}=\mathbb{C}^{n}$, where $J$ is the usual complex structure on $\mathbb{C}^{n}$ (cf. [21] for more details). $\mathbb{G}$ will be endowed with the induced Euclidean structure and the corresponding Lebesgue measure which will be denoted by $\lambda_{\mathbb{G}}$.

Let $\alpha>0$ be a positive real number. According to [22, 23], the Orlicz space $L \log ^{n+\alpha} L\left(d \lambda_{\mathbb{G}}\right)$ consists of $\lambda_{\mathbb{G}}$-measurable functions $g$ defined on $\Omega \cap \mathbb{G}$ such that

$$
\int_{\Omega \cap \Gamma} \frac{|f|}{\lambda} \log ^{n+\alpha}\left(1+\frac{|f|}{\lambda}\right) d \lambda_{\mathbb{G}}<\infty, \quad \text { for some } \lambda>0 .
$$

On the space $L \log ^{n+\alpha} L(d \mu)$, we define the norm

$$
\begin{aligned}
& \|f\|_{L \log ^{n+\alpha} L} \\
& \quad:=\inf \left\{\lambda>0 ; \int_{\Omega} \frac{|f|}{\lambda} \log ^{n+\alpha}\left(e+\frac{|f|}{\lambda}\right) d \lambda_{\mathbb{G}}<1\right\} .
\end{aligned}
$$


The dual space to $L \log ^{n+\alpha} L$ is the exponential class $\operatorname{Exp} L^{1 / n+\alpha}$; that is, the vector space

$$
\begin{aligned}
\operatorname{Exp} L^{1 / n+\alpha}:= & \{f: \Omega \longrightarrow \overline{\mathbb{R}} ; \exists \lambda>0: \\
& \left.\int_{\Omega} \exp \left(\left(\frac{|f|}{\lambda}\right)^{1 / n+\alpha}\right)-1 d \lambda_{\mathbb{G}}<\infty\right\}
\end{aligned}
$$

equipped with the norm

$$
\begin{aligned}
& \|f\|_{\operatorname{Exp} L^{1 / n+\alpha}} \\
& :=\inf \left\{\lambda>0 ; \int_{\Omega}\left(\exp \left(\left(\frac{|f|}{\lambda}\right)^{1 / n+\alpha}\right)-1\right) d \lambda_{\mathbb{G}}<1\right\} .
\end{aligned}
$$

Then we have the following Hölder inequality:

$$
\left|\int_{\Omega} f g d \lambda_{\mathbb{G}}\right| \leq C_{n, \alpha}\|f\|_{L \log ^{n+\alpha} L}\|g\|_{\operatorname{Exp} L^{1 / n+\alpha}}
$$

for $f \in L \log ^{n+\alpha} L$ and $g \in \operatorname{Exp} L^{1 / n+\alpha}$, where $C_{n, \alpha}>0$ is a positive constant depending only on $n$ and $\alpha$. By a simple computation, we have

$$
\|1\|_{\operatorname{Exp}^{1 / n+\alpha}(K)}=\frac{1}{\log ^{n+\alpha}\left(1+1 / \lambda_{\mathbb{G}}(K)\right)} .
$$

Corollary 14. Let $\mu=\nvdash_{\Omega \cap G} g \lambda_{\mathbb{G}}$ be a measure with nonnegative density $g \in \operatorname{Llog}^{n+\alpha} L(\Omega \cap \mathbb{G})$. Then there exists a unique bounded function $\varphi \in \mathscr{F}^{a}(f) \cap L^{\infty}(\Omega)$ such that $\left(d d^{c} \varphi\right)^{n}=\mu$ and

$$
0 \leq f-\varphi \leq C\|g\|_{L \log ^{n+\alpha} L}^{1 / n}
$$

where $C>0$ only depends on $n, \alpha, \Omega$, and $\mathbb{G}$.

Proof. We claim that there exists a constant $C>0$ such that

$$
\begin{array}{r}
\mu(K) \leq\left(C\|g\|_{L \log ^{n+\alpha} L}^{1 / n}\right)^{n} \operatorname{cap}_{\Omega}^{(\alpha+n) / n}(K), \\
\forall \text { compact } K \subset \Omega .
\end{array}
$$

Indeed, Hölder's inequality and inequality (66) yield

$$
\begin{array}{r}
\mu(K) \leq\|g\|_{L \log ^{n+\alpha} L} \frac{1}{\log ^{n+\alpha}\left(1+1 / \lambda_{\mathbb{G}}(K)\right)}, \\
\forall \operatorname{compact} K \subset \Omega .
\end{array}
$$

By [21] we have

$$
\lambda_{\mathbb{G}}(K) \leq C \exp \left(-\frac{1}{\operatorname{cap}_{\Omega}^{1 / n}(K)}\right), \quad \forall \text { compact } K \subset \Omega,
$$

where $C>0$ is a constant which depends only on $\Omega$ and $\mathbb{G}$.
Inequality (66) follows by combining (67) and (68). Then we apply Theorem 13 with

$$
\varepsilon(x)=C\|g\|_{L \log ^{n+\alpha} L} C\|g\|_{L \log ^{n+\alpha} L}^{1 / n} e^{-\alpha x / n},
$$

which yields

$$
\begin{aligned}
0 & \leq f-\varphi \leq e \int_{0}^{x} \varepsilon(t) d t+e \varepsilon(0)+\mu(\Omega)^{1 / n} \\
& \leq C\|g\|_{L \log ^{n+\alpha} L}^{1 / n} .
\end{aligned}
$$

\section{Conflict of Interests}

The author declares that there is no conflict of interests regarding the publication of this paper.

\section{Acknowledgment}

The author wishes to thank the referee for his careful reading and for his remarks which helped to improve the exposition.

\section{References}

[1] E. Bedford and B. A. Taylor, "The Dirichlet problem for a complex Monge-Ampère equation," Inventiones Mathematicae, vol. 37, no. 1, pp. 1-44, 1976.

[2] E. Bedford and B. A. Taylor, "A new capacity for plurisubharmonic functions," Acta Mathematica, vol. 149, no. 1-2, pp. 1-40, 1982.

[3] S. Kołodziej, "The range of the complex Monge-Ampère operator," Indiana University Mathematics Journal, vol. 43, no. 4, pp. 1321-1338, 1994.

[4] S. Kołodziej, "The complex Monge-Ampère equation," Acta Mathematica, vol. 180, no. 1, pp. 69-117, 1998.

[5] S. Kołodziej, "The complex Monge-Ampère equation and pluripotential theory," Memoirs of the American Mathematical Society, vol. 178, no. 840, 2005.

[6] R. Czyż, "The complex Monge-Ampère operator in the Cegrell classes," Dissertationes Mathematicae, vol. 466, 83 pages, 2009.

[7] U. Cegrell, "Pluricomplex energy," Acta Mathematica, vol. 180, no. 2, pp. 187-217, 1998.

[8] P. Åhag, U. Cegrell, and R. Czyż, "On Dirichlet's principle and problem," Mathematica Scandinavica, vol. 110, no. 2, pp. 235250, 2012.

[9] P. Åhag, "A Dirichlet problem for the complex Monge-Ampère operator in F(f)," Michigan Mathematical Journal, vol. 55, no. 1, pp. 123-138, 2007.

[10] P. Åhag, U. Cegrell, R. Czyz, and H. H. Pham, "Monge-Ampère measures on pluripolar sets," Journal des Mathematiques Pures et Appliquees, vol. 92, no. 6, pp. 613-627, 2009.

[11] S. Benelkourchi, "Weighted pluricomplex energy," Potential Analysis, vol. 31, no. 1, pp. 1-20, 2009.

[12] S. Benelkourchi, V. Guedj, and A. Zeriahi, "Plurisubharmonic functions with weak singularities," in Complex Analysis and Digital Geometry Proceedings from the Kiselmanfest, 2006, pp. 57-73, Uppsala Universitet, 2007. 
[13] U. Cegrell, "The general definition of the complex MongeAmpère operator," Université de Grenoble. Annales de l'Institut Fourier, vol. 54, no. 1, pp. 159-179, 2004.

[14] U. Cegrell, "A general Dirichlet problem for the complex Monge-Ampère operator," Annales Polonici Mathematici, vol. 94, no. 2, pp. 131-147, 2008.

[15] L. M. Hai and P. H. Hiep, "Some weighted energy classes of plurisubharmonic functions," Potential Analysis, vol. 34, no. 1, pp. 43-56, 2011.

[16] L. M. Hai, P. H. Hiep, N. X. Hong, and N. V. Phu, "The Monge-Ampère type equation in the weighted pluricomplex energy class," International Journal of Mathematics, vol. 25, no. 5, Article ID 1450042, 17 pages, 2014.

[17] Z. Błocki, "On the definition of the Monge-Ampère operator in $\mathbb{C}^{2}$, , Mathematische Annalen, vol. 328, no. 3, pp. 415-423, 2004.

[18] Z. Błocki, "The domain of definition of the complex MongeAmpère operator," The American Journal of Mathematics, vol. 128, no. 2, pp. 519-530, 2006.

[19] H. Le Mau, P. H. Hiep, and H. N. Quy, "Local property of the class $\mathscr{E}_{\chi, \text { loc" }}$ " Journal of Mathematical Analysis and Applications, vol. 402, no. 2, pp. 440-445, 2013.

[20] S. Benelkourchi, "Approximation of weakly singular plurisubharmonic functions," International Journal of Mathematics, vol. 22, no. 7, pp. 937-946, 2011.

[21] S. Benelkourchi, B. Jennane, and A. Zeriahi, "Polya's inequalities, global uniform integrability and the size of plurisubharmonic lemniscates," Arkiv för Matematik, vol. 43, no. 1, pp. 85112, 2005.

[22] T. Iwaniec and G. Martin, Geometric Function Theory and Non-Linear Analysis, Oxford Mathematical Monographs, The Clarendon Press, Oxford University Press, New York, NY, USA, 2001.

[23] M. M. Rao and Z. D. Ren, Theory of Orlicz Spaces, vol. 146 of Monographs and Textbooks in Pure and Applied Mathematics, Marcel Dekker, New York, NY, USA, 1991. 


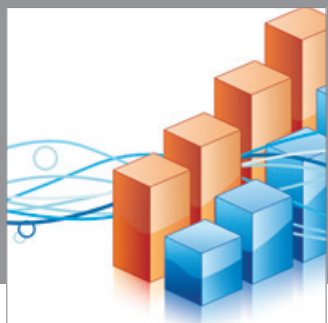

Advances in

Operations Research

mansans

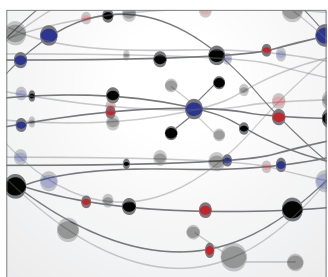

The Scientific World Journal
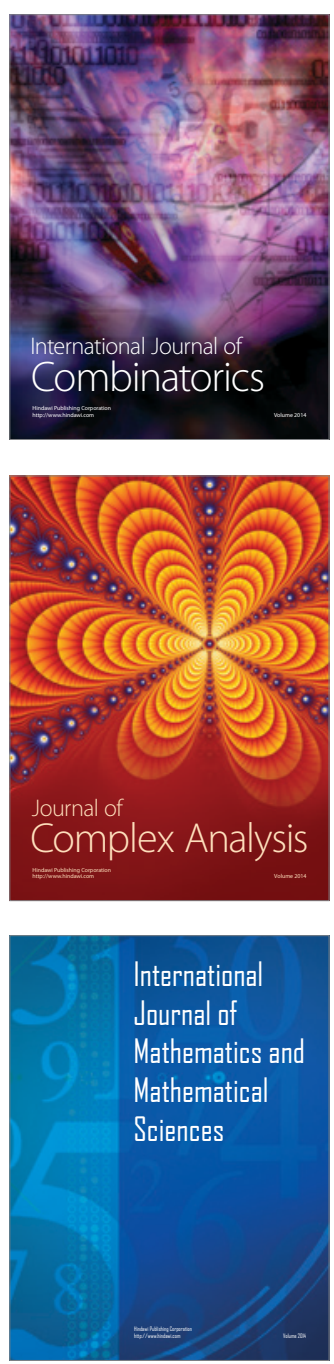
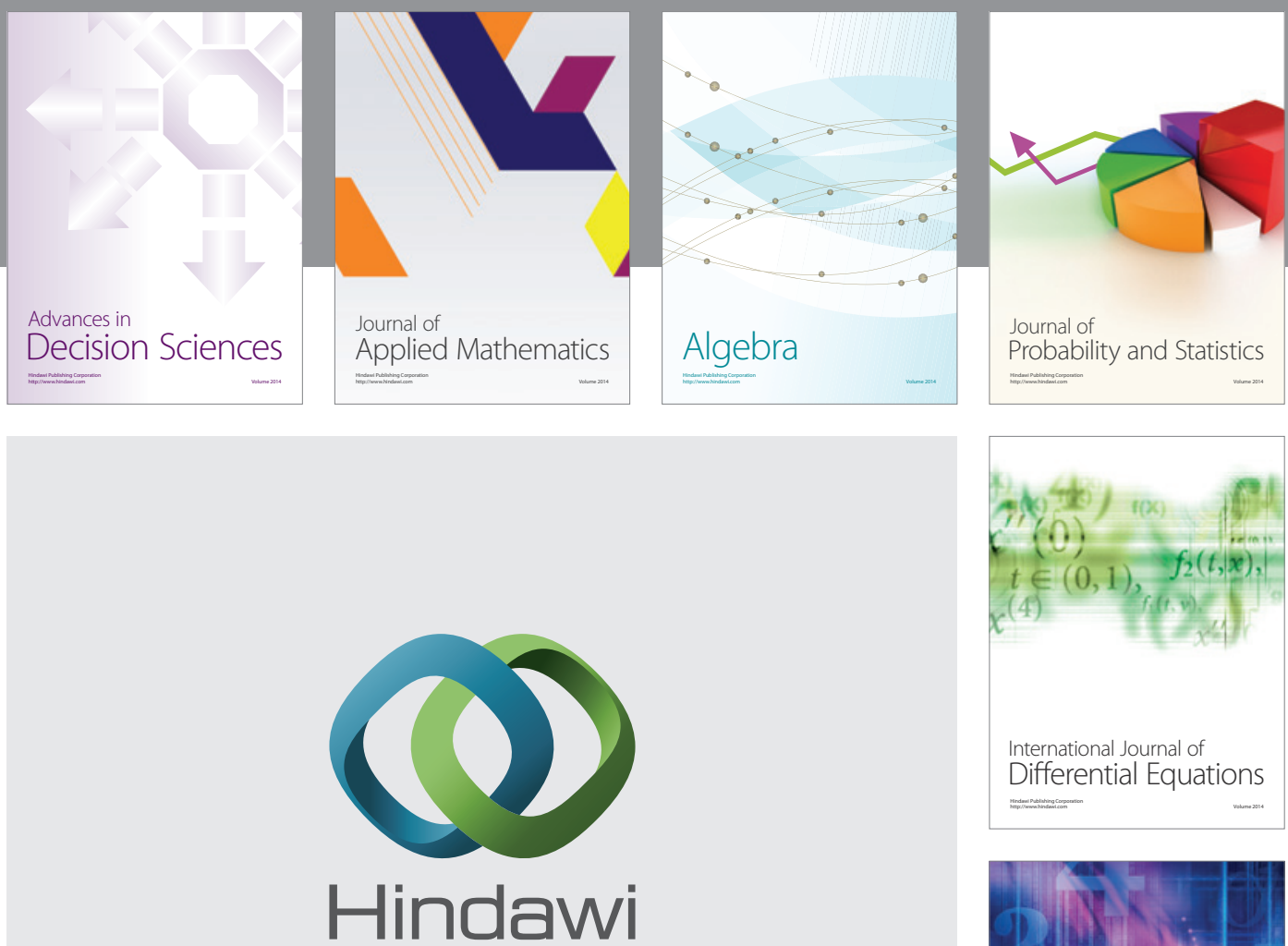

Submit your manuscripts at http://www.hindawi.com
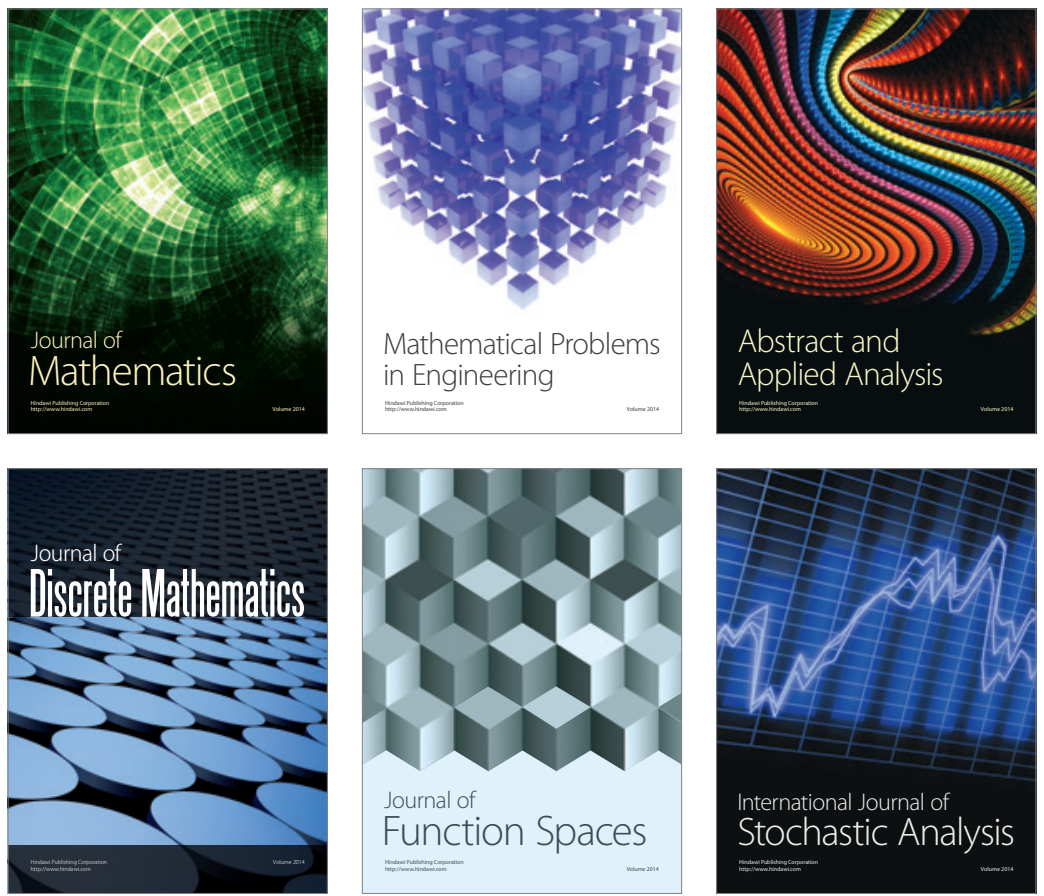

Journal of

Function Spaces

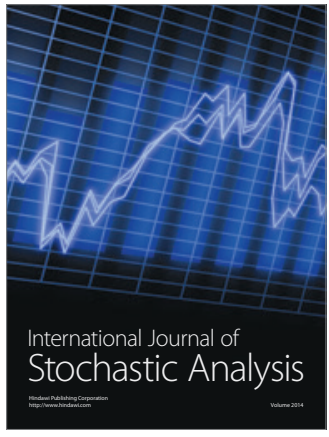

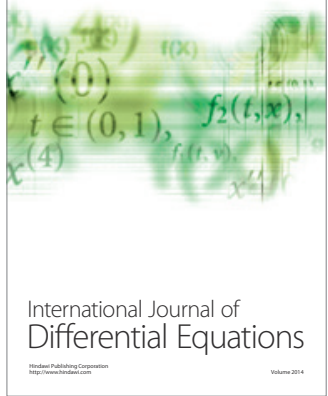
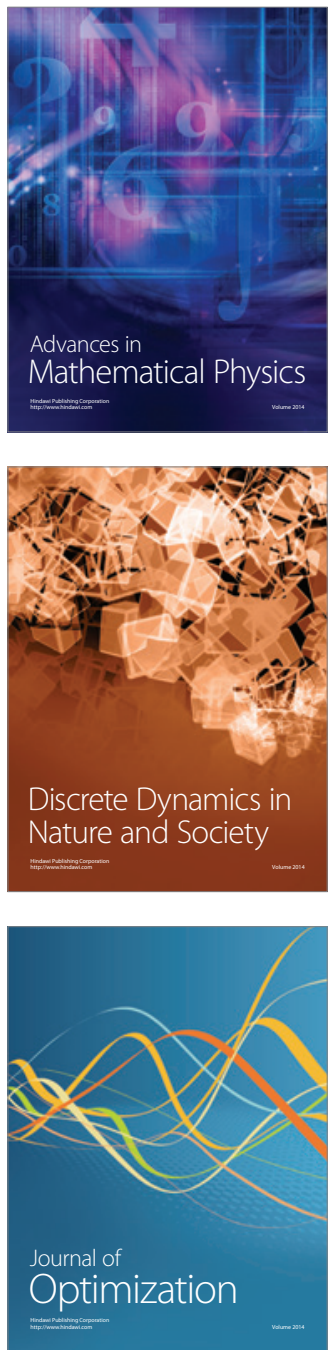